\title{
THE AGENT'S SMILE: IMPACTS OF ARTIFICIALLY GENERATED PEDAGOGICAL AGENTS ON RISK-TAKING
}

\author{
Patrick Jost \\ Department of Computer Science, \\ Norwegian University of Science and Technology, Trondheim, Norway
}

\begin{abstract}
Educators are increasingly confronted with technology-driven learning scenarios. Even before the push from the current pandemic, digital learning apps became an integrated didactic tool. Advanced computing can thereby support the digital content creation for educational courses offered on mobile platforms. Computed media content such as natural voices or generated images of real looking but non-existent persons has become easy to access and feasible to use. Particularly in exploratory learning approaches, natural environments and lifelike characters could be utilised to create compelling instructional scenarios or reality-oriented training assignments. However, there is still a limited understanding of the effects such generated lifelike persons used as Pedagogical Agents (PAs) may have on cognition and behaviour.

This study presents the findings of an international field test on the Google Play Store that investigated the effects of an artificially generated instructor's facial expression on risk-taking in a decision-making task. In the field study, an established measure of risk propensity, the Balloon Analogue Risk Task (BART), was extended to include instructions by an artificially generated lifelike persona with different facial expressions. The resulting research game for mobile phones was internationally distributed on the app store for a two-month between-subjects field experiment. The participants were instructed either by a smiling female pedagogical agent, the same agent with a neutral expression or no agent while deciding to risk further pumping up a balloon for more profit or safely collect balloons for realising a current, more modest profit. The results $(n=379)$ indicate that instructions presented by a smiling female agent reduce risk propensity in decision-making compared to instructions presented with a neutral facial expression. Instruction design considerations and experiences from the distribution of a research game on Google Play are summarised in the concluding implications.
\end{abstract}

\section{KEYWORDS}

Instructional Design, Artificial Tutors, Pedagogical Agents, Risk-Taking, Google Play Store, Field Experiment, Serious Game

\section{BACKGROUND AND RESEARCH OBJECTIVES}

Remote and mobile learning are increasingly important scenarios in educational practice. The required digital content creation for educational apps stretches from illustrations to instructional videos and virtual tutor characters (i.e. pedagogical agents) (Clark \& Mayer, 2016). While publishing apps on mobile stores has become feasible even in small-scale education scenarios, designing instructional content adequate for mobile and distributed learning remains challenging. It is well known that content presentation and interaction influence cognition and can, for example, create extraneous cognitive load (Jost et al., 2020).

These influences can impact learning gain and are critical to regard when designing for learner evaluation. Reduced personal interaction between educators and students in remote/mobile learning further complicates the design of instructions and assignments.

Advanced computing may help create educational content for mobile apps by generating visually realistic learning scenarios or lifelike instructors who act as mentors, facilitators, or examiners. Generative Adversarial Networks (GAN), a machine learning technique, has been developed intensively during recent years and can now create photorealistic pictures of non-existing persons with different facial expressions and lifelike video portraits (Liu et al., 2021). Moreover, services offer generative APIs and royalty-free, pre-generated portraits (e.g. Www.generated.photos) that can be used as pedagogical agent (PA) in educational or research-oriented 
mobile apps at no charge. Using such artificially generated media allows for learning or training apps with a lifelike instructional PA without copyright concerns or photo shootings. In addition, the parametric flexibility in creating specific facial expressions provides numerous opportunities to emphasise instructions, highlight critical pitfalls in trainings or create a more sympathetic and engaging learning environment.

However, there is currently limited insight into how such lifelike-generated personas impact cognition or behaviour. A better understanding of these influences is needed to realise the full potential of generated PAs for designing instructional apps. This study, therefore, investigates how the facial expressions of a generated lifelike PA affect risk-taking in a decision-making task to inform instructional design for educational and research mobile apps.

\subsection{Pedagogical Agents in Digital Learning and Examination Scenarios}

Commonly understood by a PA is a visually represented character used in digital learning to instruct and guide learners (Veletsianos \& Russell, 2014). The impact of pictured (Khan \& Sutcliffe, 2014), animated (van der Meij et al., 2015) and voiced PA (Domagk, 2010) has been researched in a broad area of instructional applications. Thereby PAs have been found to improve learning outcome and performance (e.g. Atorf et al., 2019; Schroeder et al., 2013). Researchers also investigated how realism in depicting the PA impacts learning gain and found that a more realistic PA representation leads to higher learning gains (Salehi \& Teymouri Nia, 2019). Moreover, studies found low or no impact on extraneous cognitive load when instructing by PA through gestures (Davis, 2018) or in multiple-choice scenarios (Schroeder, 2017). The influences of PAs in multiple-choice decision-making are of particular importance in the mobile learning context. Typing longer texts on the phone is tedious, and learner assessment is therefore often designed with choice dialogues or quizzes presented by a PA (e.g. Oyelere et al., 2018). In this regard, studies have found that risk propensity is influencing decision-making in multiple-choice exams (Biria \& Bahadoran, 2015; Yang \& Tackie, 2016). The reported findings suggest that multiple-choice exams are disadvantageous for students with lower risk propensity and suspect a gender bias preferring male students.

Studies applying PA instructions have also reported various impacts on emotions and behaviour. Dinçer and Doğanay (2017) found positive effects on motivation when applying instructive PAs in teaching software skills. Additionally, some researchers found gender-specific motivational benefits on math learning from animated (Arroyo et al., 2011) and cartoon-illustrated (Bringula et al., 2018) PA instructors with female students profiting more than male. Kahn and Sutcliffe (2014), on the other hand, investigated the influences of PA attractiveness on persuasion and found a positive correlation. The influences of different PA facial expressions have been explored on the notion of human mimicry (Chartrand \& Van Baaren, 2009) and emotional contagion (Hatfield et al., 1993). Tsai and colleagues (2012) have looked at smiling virtual agents in association with decision-making and concluded that the detected emotional contagion effect is repealed by strategic reasoning.

Investigations on PA smile frequencies/timings by Krämer et al. (2013) further confirmed emotional mimicking and found it also occurs when people were not consciously aware of the PA facial expression. DeMelo et al. (2012) analysed decision-making in a negotiating process with a PA with different facial expressions. Their results indicated that people were more likely to accept a deal when the PA expressed joy. Liew et al. (2016) researched the influences on learner motivation between neutral and smiling PA portraits in virtual learning environments. They concluded that the smile of the PA was perceived as non-genuine by the participants and thus affected motivation negatively.

However, PA visualisations generated by GAN, which are virtually indistinguishable from natural persons/photographs, have only recently become available for research in instructional scenarios. A recent study examining the influences of such lifelike PA portraits showed that persons instructed by a smiling male PA took higher risks in their decisions than those instructed by a smiling female PA (Jost, 2020c). This present study extends on these results and further investigates the suggested impact of lifelike female PA instructors on decision-making.

\subsection{Research Objectives}

The focus of this research is to investigate how the smiling and non-smiling of a lifelike female PA might influence risk-taking in a decision-making task. Using an established behavioural measure of risk-taking, the Balloon Analogue Risk Task (BART) (Lejuez et al., 2002), and extending it with instructions from the generated PA, 
a mobile research game is created and deployed in an international field test in the Google Play Store. The research objectives of this field study were thereby twofold:

1. Investigating the influences of artificially generated smiling and non-smiling female instructor personas on risk propensity in a decision-making exercise to inform instructional design practice.

2. Providing practical insights into improving the design of games for research purposes distributed via mobile app stores.

\section{EMPIRICAL RESEARCH APPROACH}

\subsection{Creating and Configuring the Research Game for Mobile Phones}

The creation of the mobile research balloon task closely followed the conduct and constructs of the original BART experiment (Lejuez et al., 2002). The BART presents a setting where participants can pump up a balloon. For each pump, a fixed amount of virtual currency (e.g. five dollars) is added to a temporary account, but only when the participant decides to collect the balloon, the amount gets transferred to a permanent account. If the user decides to take a higher risk, further pump up the balloon, and it explodes in the process, the accumulated temporary amount is lost, and the next balloon starts.

Since the initial BART experiment, the validity of the constructs for assessing individual risk propensity has been demonstrated in numerous studies concerning decision-making in mobile and other scenarios (Lauriola et al., 2014; Li et al., 2020; MacLean et al., 2018). Similarly, the author's prior field study on generated female/male PA influences employed the BART constructs by creating a mobile app with a research game framework (Jost, 2020a, 2020c). The same framework - the Quest Game-Frame - with scripted components for the Unity game engine (unity.com) was thus used to create and configure the mobile research game for this study to allow for comparison of results and research process.

The provided logging modules were utilised to protocol the BART risk parameters (see section 2.2). Data was stored in a database under the researchers' authority in a GDPR-compliant manner without personal references using a participation token and secure https connection. Informed consent form and privacy policy were shown as an introductory screen before the game. Participants were asked to give active consent and indicate age group and gender via drop-down before proceeding to the game task. The BART research scenario was designed as described by Lejuez et al. (2002), using the same text/terminology, interaction buttons and general screen layout. Instructions were presented as in the original experiment with only a text without a PA in experimental condition $A$; condition $B$ was extended by a generated non-smiling, female PA instructor. In condition $C$, the same artificial instructor persona was shown with a smiling facial expression (Figure 1).
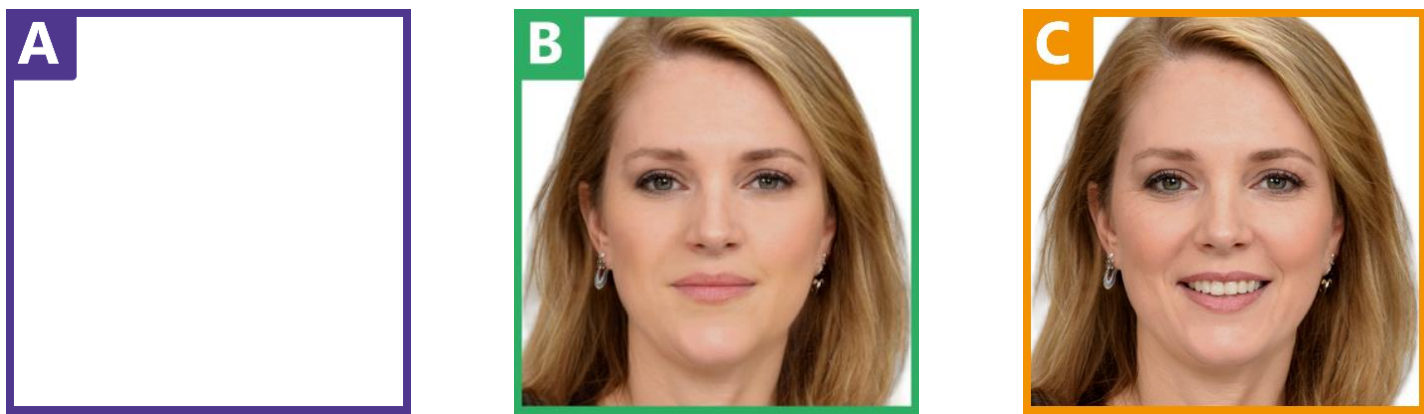

Figure 1. Experimental conditions: A - no PA; B - generated non-smiling, female PA; C - generated smiling female PA

The female PA was randomly selected on www.generated.photos/faces where GAN generated, non-existent person portraits are offered free of charge with attribution. Since the offered collection of over 2.5 million generated portraits included "beautified" portraits, a selection filter was set to include only natural, front-facing portraits of adults. As the previous study on male/female PA influences was conducted with a PA of white ethnicity, this pre-selection was also made to maintain comparability. With these inclusion criteria set, the first 
portrait of a female persona featuring both a neutral and joyful expression was selected as PA for the study (Figure 1). The PA portraits were included in the mobile game to instruct the BART rules and report the score in the decision-making exercise (Figure 2).
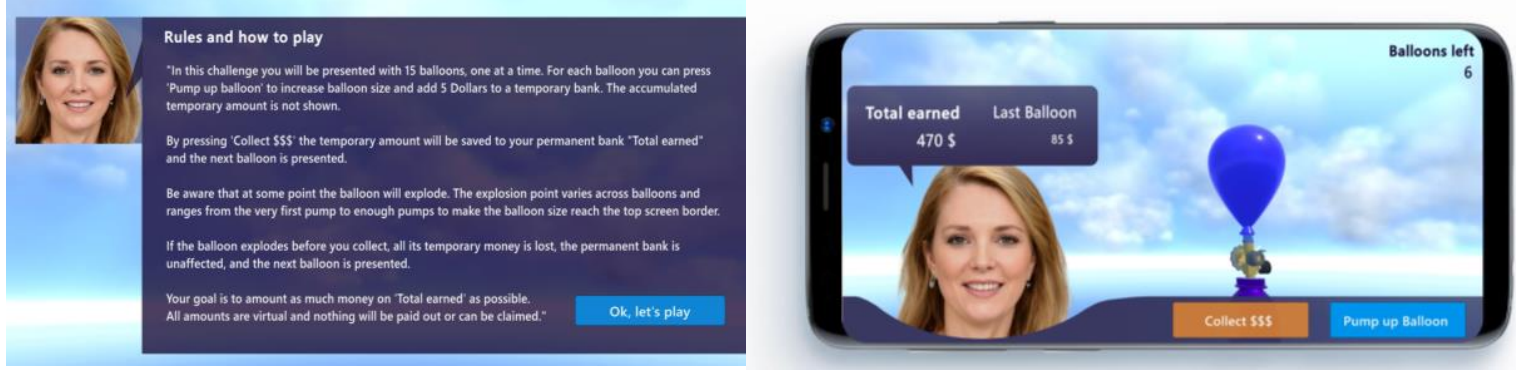

Figure 2. Mobile instruction app with generated smiling female pedagogical agent (condition C)

Following the preceding study (Jost, 2020c), the task featured 15 balloons to pump up with a pump range between 1 and 64. As in the original BART trials, the average breaking point (i.e. on which pump the balloon explodes) for each balloon was pre-calculated to improve the validity of the experiment. Integer series with values 1 to 64 were shuffled until a collection of 15 series was found with the exact breakpoint average of 32 . The balloon would pop at the random position of number 1 in each of the series. However, opposed to the BART laboratory experiment, the mobile game could be played as much as players liked. After each trial with 15 balloons, the players could immediately retry the task or finish for now and come back at a later point to play again. Players were informed that the balloon may burst at each pump and that the maximum size to which the balloon can be inflated is the top border of the screen. For assessing PA influences between the three conditions, only each participant's first trial was evaluated in the between-subjects research design. Logging was used to ensure an even distribution of the experimental conditions by assigning the next participant the variant with the lowest number of participations. The assigned condition was kept the same for the participant also in case of retries. The created mobile research game was published internationally on the Google Play Store for a two-month field test.

\subsection{Research Design, Hypothesis and Data Collection}

The game was configured to measure the BART risk constructs, mean pumps before collection/explosion and total collected/exploded balloons (Figure 3). Lejuez et al. (2002) found that each of the constructs represents valid measures of risk propensity. However, the total collected/exploded balloons are mutually exclusive, meaning a collected balloon cannot be exploded, and an exploded balloon cannot be collected. The two measures can, therefore, essentially be seen as representing a quota of 15 balloons.

Experimental condition

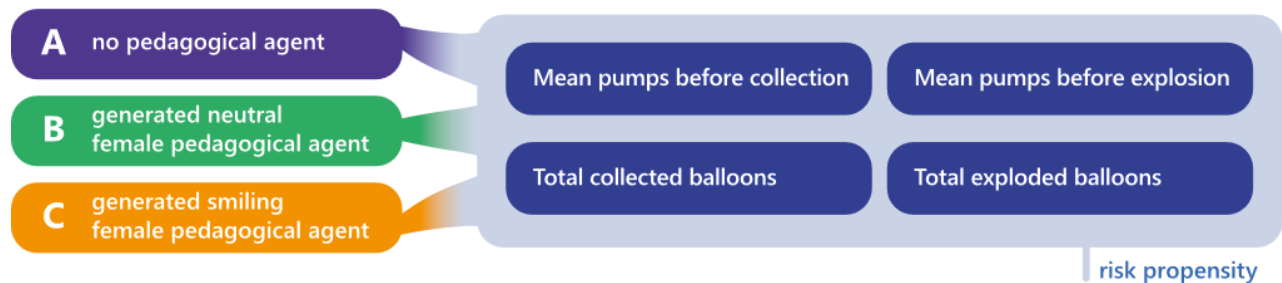

Figure 3. Experimental design for investigating PA expression influences with the mobile balloon risk task

The three independent experimental conditions to investigate influences on risk propensity were designed to show either no PA as in the original BART (condition A), the generated non-smiling female PA (condition $B$ ) or the smiling female PA (condition $C$ ) as instructor and announcer of progress (score) in the task. It was hypothesised that there would be a difference in the conditions since the preceding study had found effects when displaying a female instructor PA (Jost, 2020c). Accordingly, the null hypothesis for the field study was established as: 
$H_{0:} \quad$ 'There are no significant differences in the measured BART risk propensity constructs when instructing participants with either a smiling, non-smiling or no generated female persona visualisation.'

The mobile game was internationally published in English on the Google Play Store. For the field study, the app ran two months from February to the same day in April 2021. An international Google Ad was run with an increase of budget compared to the prior study from $0.5 €$ /day to $4.7 € /$ day as suggested by the Google algorithm. The app was presented in the store as a research game that gives some insight into individual risk propensity compared to other players. In contrast to the prior study, the average score and the total high score of all players were shown after each trial run of 15 balloons before participants could choose to either quit or play again. The Google Play Store analytics showed that the research game was compatible with 17807 different devices and offered in the store of 177 countries. Users could only proceed to the balloon game exercise after giving active, informed consent and stating age-group and gender in the introductory screen. Contrary to the previous study, reporting the country where the game was played was no longer asked from the player, as this data was available through Google Play Store analytics. The BART risk constructs were only stored in the database after a completed game run with 15 balloons either collected or exploded. The collected data were statistically analysed after the field test period with IBM SPSS Statistics 27.

\section{RESULTS}

\subsection{Field Experiment Participation}

Throughout the two months, 7595 persons from 83 different countries installed the research game. About $48 \%$ identified themselves as female participants, $40 \%$ as male and $12 \%$ indicated their gender as other. However, only $5 \%$ converted to valid participation in the balloon decision exercise (Table 1). The majority dropped out before giving informed consent. About $48 \%$ of the recorded balloon test results were repeated trials from the same person identified by the participation token.

Table 1. Participant distribution of the two-month field experiment

\begin{tabular}{lccccc}
\hline & $n$ & Quota & $\begin{array}{c}A \\
\text { (no PA) }\end{array}$ & $\begin{array}{c}\text { B } \\
\text { (neutral PA) }\end{array}$ & $\begin{array}{c}C \\
\text { (smiling PA) }\end{array}$ \\
\hline Installations from Google Play Store & 7595 & $100 \%$ & - & - & - \\
Unique participations & 464 & $6 \%$ & 149 & 157 & 158 \\
Valid participations & 379 & $5 \%$ & 115 & 136 & 128 \\
\hline
\end{tabular}

Eventually, 464 records were unique/first trial balloon test results. From these unique trials, 85 were excluded as they either had less than 20 pumps (61 entries) during the whole task or the average time between each pump decision was 0 (14 entries) or over 20 seconds (10 entries). These cases were identified as invalid as the participants either rushed through the steps without making a serious effort or were considerably distracted.

\subsection{BART Risk Constructs}

The requirements analysis indicated that the recorded valid data were not normally distributed and showed heteroscedasticity of variances. However, analysis of variance (ANOVA) is considered robust against the normality assumption for large sample sizes $(n>30)$ (Field, 2017). Particularly when distributions show only moderate skewness and kurtosis between -1 and 1 (Blanca et al., 2017). Furthermore, as Field (2017) pointed out, Welch's $F$ test does not assume equal variances while having greater statistical power than non-parametric alternatives. Following these indications, Welch's ANOVA was conducted for analysis $(a=0.05)$. 
Table 2. BART results

\begin{tabular}{lrrr}
\hline & \multicolumn{1}{c}{$\begin{array}{c}\text { A } \\
\text { (no PA) }\end{array}$} & $\begin{array}{c}\text { B } \\
\text { (neutral PA) }\end{array}$ & $\begin{array}{c}\text { (smiling PA) } \\
\text { Mean collected balloons }\end{array}$ \\
\hline 95\% CI lower / upper & 10.9 & ${ }^{*} 10.4$ & ${ }^{*} 11.5$ \\
Mean exploded balloons & $10.3 / 11.4$ & $9.8 / 11.1$ & $11.0 / 12.0$ \\
95\% CI lower / upper & 4.1 & $* 4.6$ & $* 3.5$ \\
SE collected \& exploded balloons & $3.6 / 4.7$ & $3.9 / 5.2$ & $3.0 / 4.0$ \\
Mean pumps before collection & 0.3 & 0.3 & 0.3 \\
SE pumps before collection & 4.5 & 4.8 & 4.9 \\
Mean pumps before explosion & 0.5 & 0.5 & 0.5 \\
SE pumps before explosion & 25.4 & 24.5 & 24.4 \\
\hline
\end{tabular}

* Significant differences between conditions B and C, $p=.034$

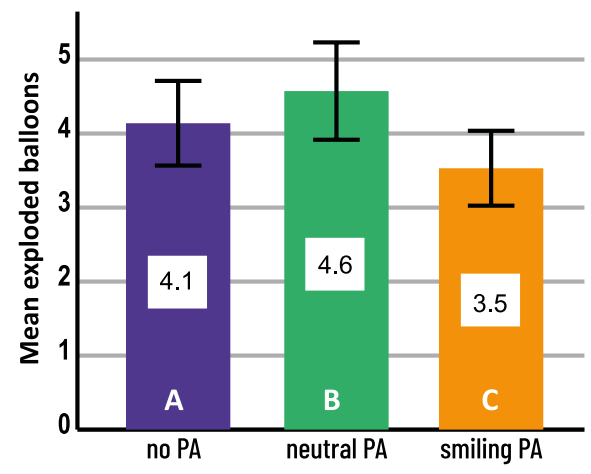

Figure 4. Significantly less exploded balloons with smiling female PA [+/-2 SE]

Analysis showed no significant differences between the instruction scenarios for mean pumps before collection, $F(2,249.9)=0.189, p=.828$ and mean pumps before explosions, $F(2,249.0)=0.439, p=.645$. However, the balloon test outcome differed significantly between the three instruction conditions regarding collections/explosions of balloons, $F(2,247.9)=3.35, p=.037$. Pairwise comparisons with Games-Howell post-hoc procedure was then conducted to determine which of the A, B and C scenarios resulted in significantly different means of collected/exploded balloons. The results revealed that participants decided differently in the $\mathrm{B}$ and $\mathrm{C}$ conditions where they were instructed by the neutral or smiling generated female PA. As displayed in Table 2 and Figure 4, participants were on average more inclined to collect balloons than pump them further and risk explosion when instructed by the smiling PA compared to the non-smiling PA (mean difference exploded balloons $=-1.04 ; 95 \% \mathrm{CI}=-2.02,-0.06 ; p=.034$ ) with a small to medium effect size of Cohen's $d=0.3$. Consequently, statistical analysis indicates to reject $H_{0}$.

\section{DISCUSSION}

\subsection{Interpretation of PA Influences on Instructional Design}

When interpreting the results regarding the first research objective about the impact of an artificial PA's facial expressions, the study disclosed mainly three insights to inform instructional design.

First, the outcome confirms prior findings that instructions presented by a lifelike generated PA can impact taking chances in decision-making tasks. As previously found with male/female PA instructors (Jost, 2020c), differences in participants' decision making were also found when a PA with a neutral or a smiling facial expression instructed them. While participants inflated the balloons on average to a similar extent, the group instructed by the smiling PA collected the balloons more frequently and thus risked less of the balloons exploding.

Second, the impact of PA facial expressions could be utilised as subtle nudging mechanism in digital learning scenarios. This finding on facial expression influence supports the results of Kahn and Sutcliffe (2014), who found that more attractive PA pictures are more persuasive. In the BART scenario, the collecting action that grants the money could be seen as offer from the PA, which was more often accepted by the participants shown the smiling PA. On the other hand, it is also possible that the participants were subconsciously in a more positive mindset due to emotional contagion originating from the smiling PA (Krämer et al., 2013), resulting in more active avoidance of explosions. Further research is required to investigate this distinction and the nature of the more cautious decision-making when instructed by a smiling PA. In any case, the subtle influences from PA expressions on risk-taking present various instructional design opportunities. Educational content creators could, for example, use generated PA instructors with sympathetic expressions that support more cautious practices in training applications. 
Third, the emotional impact of PA facial expressions needs to be considered when creating instructional apps that assess learner progress or include research objectives. Generated lifelike PA also present an opportunity for designing exploratory or game-based learning where they could be applied in instructional, assessment or motivational functions. However, the reported PA influences require careful consideration when assessing learner progress. Results from dialogue prompts presented by a generated PA may be confounded by different PA facial expressions or gender, which is of particular concern when also including research-oriented assessment (Jost, 2020b).

\subsection{Implications for the Creation of Mobile Research Games}

In general, utilising the Quest Game-Frame facilitated the iterative adaption of the research scenario and republishing the research game to the Google Play Store, which could be done within a couple of hours. In relation to the second research objective, the study confirmed and highlighted some design considerations from practice for the creation of mobile research games.

First of all, consent to data collection discourages most users from engaging in a research game. Only 5\% of installations converted to valid participations. Most users that installed the app did not engage in playing but dropped out at the consent page even though it was simplified for this study. Thus, researchers must consider a high conversion drop-out quote when planning GDPR conform field trials with a mobile game.

Next, advertising the mobile app with an online ad campaign is required but also feasible and can be controlled by budget increase. The preceding field trial employed a Google Ad campaign with a budget of 0.5 $€ /$ day that generated 20.6 installations per day. Increasing the budget to $4.7 €$ /day generated 128.7 daily installations. An approx. 10-fold higher budget led to a 6-fold higher installation volume. Thus, raising the ad budget effectively controls the participation rate when also considering the drop-out quote.

Finally, showing participants a comparative score of other players can considerably increase the replay rate. If the research includes repetitive trials, it can be supported by showing participants the average score and current high score combined with a retry button after each round of play. Providing this information to players led to an increase in replays in this field study compared to a preceding one, from $22 \%$ to $48 \%$.

\subsection{Limitations and Further Research Trails}

This study and its findings are limited in several aspects that must be addressed. The Google Play Store was used to distribute and advertise the mobile game internationally in 177 countries. However, the interface language was English only. As the research game was played in 83 countries, several people with limited understanding of the instructions will likely have participated in the trials. Moreover, influences originating from different cultural backgrounds were not considered since only a PA of white ethnicity was pictured. Another aspect to consider for all field studies is the limited control of the context of play. Although extreme values could be identified by time logging, other more subtle contextual influences and distractions could have contributed to the group differences. Further research on influences from artificially generated PAs should include more controllable research designs such as laboratory studies. Psychophysiological measurements, including skin conductance or eye-tracking, could thereby provide further insight into how features of a generated PA affect decision-making.

\section{CONCLUSION}

The reported result from the two-month field study confirmed previous studies that found influences from pedagogical agents on decision-making. Participants took a less risky approach in the mobile game based on the balloon risk task if a generated smiling PA instructed them compared to a non-smiling variant. The generation of lifelike personas as portraits and video content offers vast opportunities for instructional design in education and research. Knowing the impact of facial expressions allows adapting tutor design to support pedagogical and research-oriented strategies. The findings suggest that sympathetic generated female PAs could be utilised in digital training applications to unobtrusively support more cautious decision-making where it is helpful. Moreover, the study demonstrated the utility of a mobile game as research instrument. The international field experiment on an app store was feasible and controllable while creating valuable insight for 
instructional design of artificial PA instructors. However, the high drop-out rate due to informed consent and other field study constraints requires careful planning of research conduct. Ultimately, artificially generated PAs and their features need to be further investigated for their potentials. Therefore, field studies should be complemented with controlled laboratory experiments to learn more about how generated lifelike PAs can be used beneficially for education and research.

\section{ACKNOWLEDGEMENT}

This research was supported by the Research Council of Norway (Norges Forskningsråd) by funding the IKTPLUSS project ALerT, \#270969.

\section{REFERENCES}

Arroyo, I., Woolf, B. P., Cooper, D. G., Burleson, W., \& Muldner, K. (2011). The Impact of Animated Pedagogical Agents on Girls' and Boys' Emotions, Attitudes, Behaviors and Learning. 2011 IEEE 11th International Conference on Advanced Learning Technologies, 506-510. https://doi.org/10.1109/ICALT.2011.157

Atorf, D., Kannegieser, E., \& Roller, W. (2019). Study on Enhancing Learnability of a Serious Game by Implementing a Pedagogical Agent. International Conference on Games and Learning Alliance, 158-168.

Biria, R., \& Bahadoran, A. (2015). Exploring the role of risk-taking propensity and gender differences in EFL students' multiple-choice test performance. Canadian Journal of Basic and Applied Sciences, 3(05), 144-154.

Blanca, M. J., Alarcón, R., Arnau, J., Bono, R., \& Bendayan, R. (2017). Non-normal data: Is ANOVA still a valid option? Psicothema, 29(4), 552-557.

Bringula, R. P., Fosgate Jr, I. C. O., Garcia, N. P. R., \& Yorobe, J. L. M. (2018). Effects of Pedagogical Agents on Students' Mathematics Performance: A Comparison Between Two Versions. Journal of Educational Computing Research, 56(5), 701-722.

Chartrand, T. L., \& Van Baaren, R. (2009). Human mimicry. Advances in Experimental Social Psychology, 41, $219-274$.

Clark, R. C., \& Mayer, R. E. (2016). E-learning and the science of instruction: Proven guidelines for consumers and designers of multimedia learning (Fourth edition). Wiley.

Davis, R. O. (2018). The impact of pedagogical agent gesturing in multimedia learning environments: A meta-analysis. Educational Research Review, 24, 193-209. https://doi.org/10.1016/j.edurev.2018.05.002

de Melo, C. M., Carnevale, P., \& Gratch, J. (2012). The Effect of Virtual Agents' Emotion Displays and Appraisals on People's Decision Making in Negotiation. In Y. Nakano, M. Neff, A. Paiva, \& M. Walker (Eds.), Intelligent Virtual Agents (pp. 53-66). Springer. https://doi.org/10.1007/978-3-642-33197-8_6

Dinçer, S., \& Doğanay, A. (2017). The effects of multiple-pedagogical agents on learners' academic success, motivation, and cognitive load. Computers \& Education, 111, 74-100.

Domagk, S. (2010). Do pedagogical agents facilitate learner motivation and learning outcomes?: The role of the appeal of agent's appearance and voice. Journal of Media Psychology: Theories, Methods, and Applications, 22(2), 84.

Field, A. (2017). Discovering statistics using IBM SPSS statistics (5th edition). SAGE Publications.

Hatfield, E., Cacioppo, J. T., \& Rapson, R. L. (1993). Emotional contagion. Current Directions in Psychological Science, 2(3), 96-100.

Jost, P. (2020a). The Quest Game-Frame: Balancing Serious Games for Investigating Privacy Decisions. In Proceedings of the 11th Scandinavian Conference on Information Systems (SCIS2020) (pp. 1-17). Association for Information Systems (AIS). https://aisel.aisnet.org/scis2020/5/

Jost, P. (2020b). Because it is Fun: Investigating Motives of Fake News Sharing with Exploratory Game Quests. In D. G. Sampson, D. Ifenthaler, \& P. Isaías (Eds.), 17th International Conference on Cognition and Exploratory Learning in the Digital Age (CELDA 2020) (pp. 35-42). IADIS Press.

Jost, P. (2020c). Pedagogical agents: Influences of artificially generated instructor personas on taking chances. Proceedings of the Norwegian Conference for ICT-Research and Education (NIKT 2020), 4, 1-14.

Jost, P., Cobb, S., \& Hämmerle, I. (2020). Reality-based interaction affecting mental workload in virtual reality mental arithmetic training. Behaviour \& Information Technology, 39(10), 1062-1078.

https://doi.org/10.1080/0144929X.2019.1641228

Khan, R. F., \& Sutcliffe, A. (2014). Attractive agents are more persuasive. International Journal of Human-Computer Interaction, 30(2), 142-150. 
Krämer, N., Kopp, S., Becker-Asano, C., \& Sommer, N. (2013). Smile and the world will smile with you-The effects of a virtual agent's smile on users' evaluation and behavior. International Journal of Human-Computer Studies, 71(3), 335-349. https://doi.org/10.1016/j.ijhcs.2012.09.006

Lauriola, M., Panno, A., Levin, I. P., \& Lejuez, C. W. (2014). Individual differences in risky decision making: A meta-analysis of sensation seeking and impulsivity with the balloon analogue risk task. Journal of Behavioral Decision Making, 27(1), 20-36.

Lejuez, C. W., Read, J. P., Kahler, C. W., Richards, J. B., Ramsey, S. E., Stuart, G. L., Strong, D. R., \& Brown, R. A. (2002). Evaluation of a behavioral measure of risk taking: The Balloon Analogue Risk Task (BART). Journal of Experimental Psychology: Applied, 8(2), 75.

Li, X., Pan, Y., Fang, Z., Lei, H., Zhang, X., Shi, H., Ma, N., Raine, P., Wetherill, R., \& Kim, J. J. (2020). Test-retest reliability of brain responses to risk-taking during the balloon analogue risk task. NeuroImage, 209, 116495.

Liew, T. W., Zin, N. A. M., Sahari, N., \& Tan, S.-M. (2016). The effects of a pedagogical agent's smiling expression on the learner's emotions and motivation in a virtual learning environment. The International Review of Research in Open and Distributed Learning, 17(5).

Liu, M.-Y., Huang, X., Yu, J., Wang, T.-C., \& Mallya, A. (2021). Generative Adversarial Networks for Image and Video Synthesis: Algorithms and Applications. Proceedings of the IEEE, 109(5), 839-862.

https://doi.org/10.1109/JPROC.2021.3049196

MacLean, R. R., Pincus, A. L., Smyth, J. M., Geier, C. F., \& Wilson, S. J. (2018). Extending the Balloon Analogue Risk Task to Assess Naturalistic Risk Taking via a Mobile Platform. Journal of Psychopathology and Behavioral Assessment, 40(1), 107-116. https://doi.org/10.1007/s10862-017-9628-4

Oyelere, S. S., Suhonen, J., Wajiga, G. M., \& Sutinen, E. (2018). Design, development, and evaluation of a mobile learning application for computing education. Education and Information Technologies, 23(1), 467-495.

https://doi.org/10.1007/s10639-017-9613-2

Salehi, V., \& Teymouri Nia, F. (2019). Effect of levels of realism of mobile-based pedagogical agents on health e-learning. Future of Medical Education Journal, 9(2), 40-45.

Schroeder, N. L. (2017). The influence of a pedagogical agent on learners' cognitive load. Journal of Educational Technology \& Society, 20(4), 138-147.

Schroeder, N. L., Adesope, O. O., \& Gilbert, R. B. (2013). How effective are pedagogical agents for learning? A meta-analytic review. Journal of Educational Computing Research, 49(1), 1-39.

Tsai, J., Bowring, E., Marsella, S., Wood, W., \& Tambe, M. (2012). A Study of Emotional Contagion with Virtual Characters. In Y. Nakano, M. Neff, A. Paiva, \& M. Walker (Eds.), Intelligent Virtual Agents (pp. 81-88). Springer. https://doi.org/10.1007/978-3-642-33197-8_8

van der Meij, H., van der Meij, J., \& Harmsen, R. (2015). Animated pedagogical agents effects on enhancing student motivation and learning in a science inquiry learning environment. Educational Technology Research and Development, 63(3), 381-403. https://doi.org/10.1007/s11423-015-9378-5

Veletsianos, G., \& Russell, G. S. (2014). Pedagogical Agents. In J. M. Spector, M. D. Merrill, J. Elen, \& M. J. Bishop (Eds.), Handbook of Research on Educational Communications and Technology (pp. 759-769). Springer. https://doi.org/10.1007/978-1-4614-3185-5_61

Yang, Z., \& Tackie, M. (2016). Risk Preference and Student Behavior on Multiple-Choice Exams. Economics Bulletin, 36(1), 58-67. 